\title{
Generalized Pattern Spectra Sensitive to Spatial Information
}

\author{
Michael H. F. Wilkinson \\ Institute for Mathematics and Computing Science, \\ University of Groningen, PO Box 800, 9700 AV Groningen, The Netherlands
}

\begin{abstract}
Morphological pattern spectra computed from granulometries are frequently used to classify the size classes of details in textures and images. An extension of this technique, which retains information on the spatial distribution of the details in each size class is developed. Algorithms for computation of these spatial pattern spectra for a large number of granulometries on binary images are presented.
\end{abstract}

\section{Introduction}

Granulometries are powerful multi-scale tools in image analysis. They were developed to study the distribution of pore sizes in porous media [6] (for a recent review see [14]). Granulometries are ordered sets of morphological openings or closings, each of which removes image details below a certain size. By studying how the image content changes as a function of the filter's size parameter, it is possible to gain insight into the distribution of details over different size classes, often without prior segmentation of the image. The simplest way to do this is through the use of pattern spectra which show how the number of foreground pixels in the image changes as a function of the size parameter [5]. Here, size is usually defined in terms of the width of objects [7, 10], though area may be used as well [12]. This information can be used in e.g. soil analysis [11].

A drawback of the classical definition of pattern spectra is that spatial information, i.e., information about the position of components removed by each filter, is not included in a pattern spectrum. This effect is demonstrated in figure 1. All three binary images in this figure contain the same number of squares in each size category. If we apply use a granulometry consisting of openings by reconstruction with square structuring elements of width $\lambda$, the resulting pattern spectra are the same for all three images. In fact, no granulometry is capable of separating the patterns, because the only differences between the images lie in the distributions of the connected components, not in their shapes or sizes.

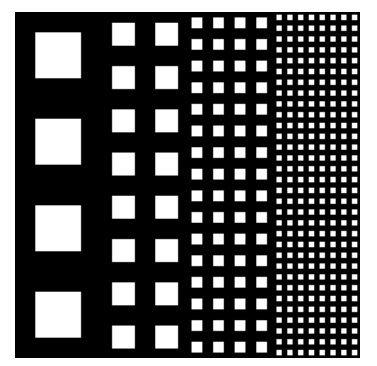

(a)

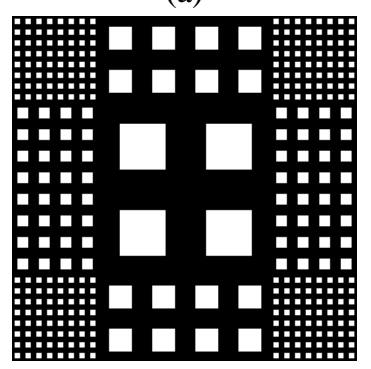

(c)

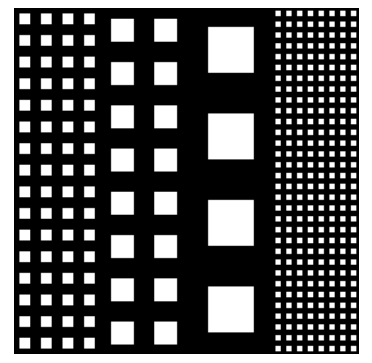

(b)

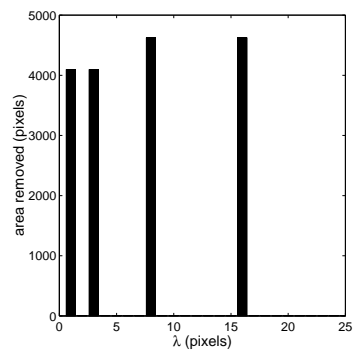

(d)
Figure 1. The insensitivity of pattern spectra to the spatial information: (a) through (c) three images consisting of squares of different sizes; (d) The pattern spectra, showing the number of foreground pixels removed by openings by reconstruction by $\lambda \times \lambda$ squares, are identical for all images.

This absence of spatial information in pattern spectra is not always a problem. When studying microscopic images of, e.g., cross-sections of metal alloy or mineral samples in which different grains or pores are embedded randomly in the material, spatial information is largely irrelevant. However, this information is essential in, e.g., the study of microbial biofilms or mats [4], where the distribution of different species of bacteria throughout the ecosystem is sought. More generally, if the distribution of details is non-random retaining spatial information may be essential for pattern analysis.

In this paper, a variant of pattern spectra is developed, 
which I will call spatial pattern spectra. These store not only the number of pixels in each size category, but also information on the distribution of these details. A subset of these methods ensure rotation, translation and scale invariance of the spatial pattern spectrum.

Besides defining spatial pattern spectra, it is shown that an algorithm based on Nacken's approach to chamfer based granulometries [10] can be adapted to spatial pattern spectra in the case of binary images.

\section{Theory}

\subsection{Granulometries}

Let binary images $X$ and $Y$ be defined as a subset of the image domain $\mathbf{M} \subset \mathbb{Z}^{n}$ or $\mathbb{R}^{n}$ (usually $n=2$ ). Grey scale images are a mapping from $M$ to $\mathbb{Z}$ or $\mathbb{R}$.

Definition 1 A binary granulometry is a set of operators $\left\{\alpha_{r}\right\}$ with $r$ from some ordered set $\Lambda$ (usually $\Lambda \subset \mathbb{R}$ or $\mathbb{Z}$ ), with the following three properties

$$
\begin{aligned}
\alpha_{r}(X) & \subset X \\
X \subset Y & \Rightarrow \alpha_{r}(X) \subset \alpha_{r}(Y) \\
\alpha_{r}\left(\alpha_{s}(X)\right) & =\alpha_{\max (r, s)}(X),
\end{aligned}
$$

for all $r, s \in \Lambda$.

Since (1) and (2) define $\alpha_{r}$ as anti-extensive and increasing, respectively, and (3) implies idempotence, it can be seen that all $\alpha_{r}$ are openings. Apart from the classical openings used by Matheron [7], other openings which qualify as granulometries include openings by reconstruction [13], area openings [12], and attribute openings [1].

\subsection{Pattern spectra and Opening Transforms}

The pattern spectrum [5] $s_{\alpha}(X)$ obtained by applying granulometry $\left\{\alpha_{r}\right\}$ to a binary image $X$ is defined as

$$
\left(s_{\alpha}(X)\right)(u)=-\left.\frac{\partial A\left(\alpha_{r}(X)\right)}{\partial r}\right|_{r=u}
$$

in which $A(X)$ is a function denoting the Lebesgue measure in $\mathbb{R}^{n}$. In the case of discrete images, and with $r \in \Lambda \subset \mathbb{Z}$, this differentiation reduces to

$$
\begin{aligned}
\left(s_{\alpha}(X)\right)(r) & =\#\left(\alpha_{r}(X) / \alpha_{r^{+}}(X)\right) \\
& =\#\left(\alpha_{r}(X)\right)-\#\left(\alpha_{r^{+}}(X)\right),
\end{aligned}
$$

with $r^{+}=\min \left\{r^{\prime} \in \Lambda \mid r^{\prime}>r\right\}$, and \#(X) the number of elements of $X$.

The opening transform [10] $\Omega_{X}$ of a binary image $X$ for a granulometry $\alpha_{r}$ is

$$
\Omega_{X}(x)=\max \left\{r \in \Lambda \mid x \in \alpha_{r}(X)\right\}
$$

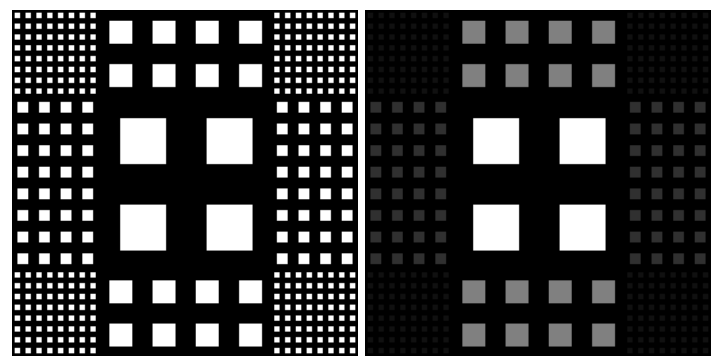

Figure 2. Opening transform with $\left\{\alpha_{r}\right\}$ as in Fig. 1: (left) original image; (right) opening transform (contrast stretched for clarity).

Figure 2 shows an example of an opening transform using the same attribute granulometry as in figure 1 . The pattern spectrum of a binary image $X$ using granulometry $\left\{\alpha_{r}\right\}$ is the histogram of $\Omega_{X}$ obtained with the same size distribution [10].

\subsection{Spatial pattern spectra}

None of the pattern spectra defined above contain any information on the spatial distribution of the details at scale $r$. They only retain the amount of detail present at that scale. This can be amended by computing some parameterization of the spatial distribution in an image $\alpha_{r}(X) / \alpha_{r+}(X)$ as a function of $r$. Let $M(X)$ be some parameterization of the spatial distribution of detail in the image $X$. The spatial pattern spectrum $S_{M, \alpha}$ is then defined as

$$
\left(S_{M, \alpha}(X)\right)(r)=M\left(\alpha_{r}(X) / \alpha_{r+}(X)\right) .
$$

An obvious parameterization of the spatial distribution is through the use of moments. Focusing on the case of 2-D binary images, the moment $m_{i j}$ of order $i j$ of an image $X$ is given by

$$
m_{i j}(X)=\sum_{(x, y) \in \mathbf{X}} x^{i} y^{j} .
$$

The spatial moment spectrum $S_{m_{i j}, \alpha}$ of order $i j$ is

$$
\left(S_{m_{i j}, \alpha}(X)\right)(r)=m_{i, j}\left(\alpha_{r}(X) / \alpha_{r^{+}}(X)\right) .
$$

For $i=0$ and $j=0$ we obtain the standard pattern spectrum. This suggests that computation of pattern moment spectra, can be performed by modification of any existing algorithm for standard pattern spectra. For each $r,\left(S_{m_{i j}, \alpha}(X)\right)(r)$ is just the moment of an image, therefore, derived parameters such as coordinates of the centre of mass, (co-)variances, skewness and kurtosis of the distribution of details at each scale can be computed easily. We can then define pattern mean spectra, pattern (co-)variance 


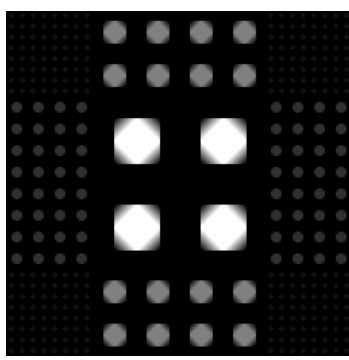

(a)

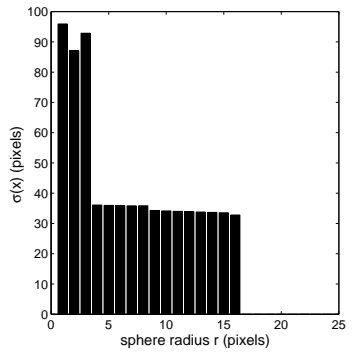

(c)

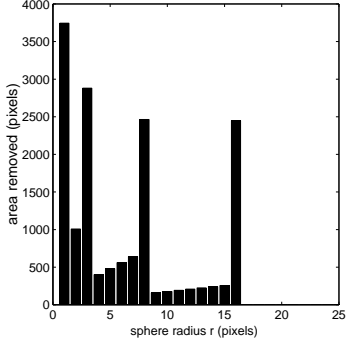

(b)

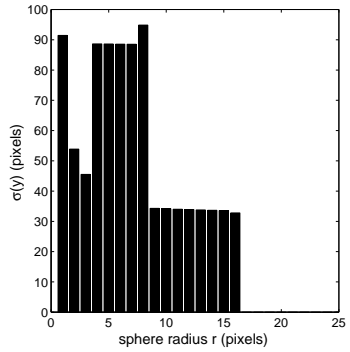

(d)
Figure 3. The opening transform using cityblock metric: (a) opening transform of Fig. 1(c), (b) pattern spectrum, (c) pattern variance- $x$ and (d) variance- $y$ spectra.

spectra, pattern kurtosis spectra, etc. The pattern mean- $x$ and variance- $x$ spectra $\left(S_{\bar{x}, \alpha}\right.$ and $\left.S_{\sigma(x), \alpha}\right)$ are defined as:

$$
\begin{aligned}
S_{\bar{x}, \alpha} & =\frac{S_{m_{10}, \alpha}}{S_{m_{00}, \alpha}} \\
S_{\sigma(x), \alpha} & =\sqrt{\frac{S_{m_{20}, \alpha}}{S_{m_{00}, \alpha}}-S_{\bar{x}, \alpha}}
\end{aligned}
$$

and likewise for $y$ and pattern skew and pattern kurtosis spectra. Note that these definitions hold only where $\left(S_{m_{00}, \alpha}(f)\right)(r) \neq 0$. For all other values of $r$ they will be defined as zero. Computing these derived spectra has the advantage of reducing the covariance between the different pattern moment spectra. Further post-processing can be done to compute central moments and moment invariant from pattern moment spectra [3, 2].

\section{An Algorithm}

In the discussion of the algorithms we will assume that the spectra are stored in an array $\mathrm{S}$ with integers $r \in \Lambda \subset \mathbb{Z}$.

Nacken [10] derived an algorithm for computation of pattern spectra for granulometries based on openings by discs of increasing radius for various metrics, using the opening transform.

He first notes that the pattern spectrum is equal to the histogram of the opening transform of the image. He proceeds by deriving an efficient algorithm for these opening transforms in the case of chamfer metrics, details of which can be found in [10]. After the opening transform has been computed, it is straightforward to compute the pattern spectrum:

- Set all elements of array S to zero

- For all $x \in X$ increment $\mathrm{S}\left[\Omega_{X}(x)\right]$ by one.

To compute the pattern moment spectrum, the only thing that needs to be changed is the way $S\left[\Omega_{X}(x)\right]$ is incremented. For a pattern moment spectrum of order $i j$ we have

- Set all elements of array $\mathrm{S}$ to zero

- For all $(x, y) \in X$ increment $\mathrm{S}\left[\Omega_{X}(x, y)\right]$ by $x^{i} y^{j}$.

Figure 3 shows the opening transform and various pattern spectra of the image of figure 1(c), for openings with spheres $B_{r}$ defined by the city-block metric. The spectra were computed according to the algorithm above. This algorithm can readily be adapted to other granulometries, simply by computing the appropriate opening transform. Two examples are attribute openings and openings by reconstruction. A binary attribute opening $\Gamma^{T}$ removes all connected foreground components from a binary image which do not meat a criterium $T$, leaving all others unaffected [1]. If we wish to use the set of attribute openings $\left\{\Gamma^{T_{\lambda}}\right\}$, with $T_{\lambda}=A(C) \leq \lambda$, with $C$ a connected set, and with $A(C)$ some increasing attribute of $C$, we can define the attribute opening transform $\Omega_{X}^{T_{\lambda}}$ as

$$
\Omega_{X}^{T_{\lambda}}(x)= \begin{cases}A\left(\Gamma_{x}(X)\right) & \text { if } x \in X \\ 0 & \text { otherwise }\end{cases}
$$

in which $\Gamma_{x}(X)$ is the connected opening of $X$ at position $x$. This is just the connected component of $X$ to which $x$ belongs, if $x \in X$, otherwise it is $\varnothing$. This transform can be computed by flood-filling each connected component by its attribute value. Once $\Omega_{X}^{T_{\lambda}}$ has been computed, the pattern moment spectra of order $i j$ can be computed in the same way as before.

A similar approach can be used for openings by reconstruction, using disc-shaped structuring elements $B_{r}$. After computing the distance map $D_{X}$ of image $X$ (for any given metric), we can compute the opening by reconstruction transform $\Omega_{X}^{B_{r}}$ as

$$
\Omega_{X}^{B_{r}}(x)= \begin{cases}\max \left\{D_{X}\left(x^{\prime}\right) \mid x^{\prime} \in \Gamma_{x}(X)\right\} & \text { if } x \in X \\ 0 & \text { otherwise }\end{cases}
$$

This can be computed by first finding the maximum value of $D_{X}$ within each connected component $C \subset X$ and then 

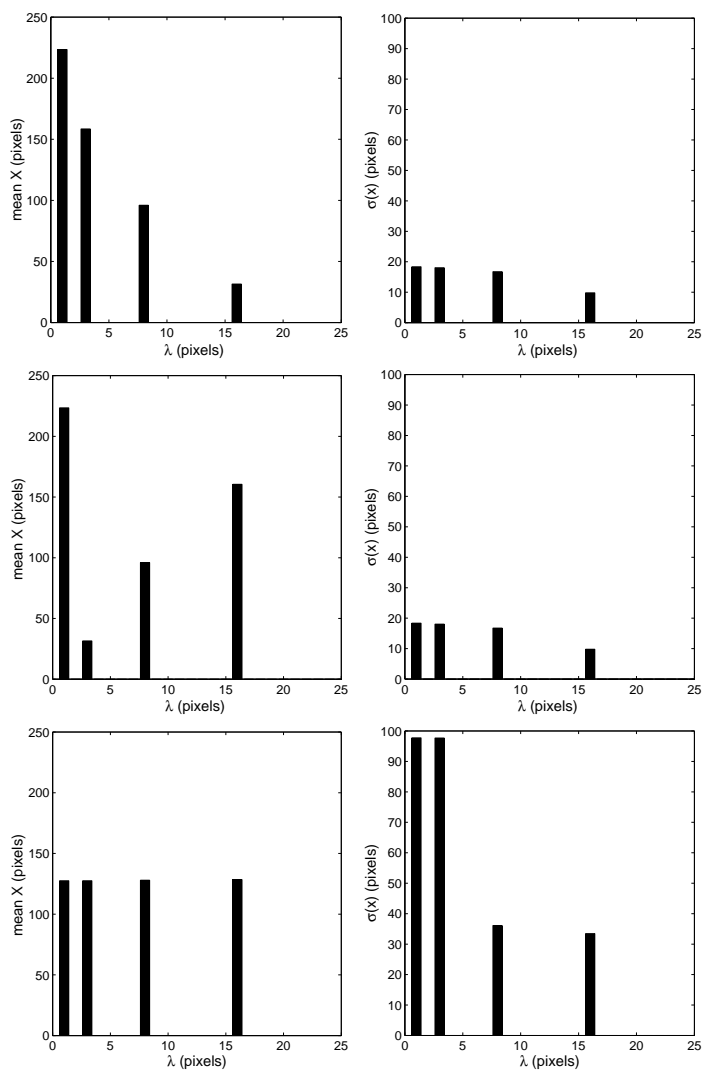

Figure 4. Pattern mean- $x$ (left) and variance$x$ (right) spectra: top to bottom rows show spectra for Fig. 1(a), (b) and (c) respectively

flood filling the component with that value. Computing the pattern moment spectra is then performed in the same way as above.

Figure 4 shows the pattern mean- $x$ and variance- $x$ spectra derived from the three images in figure 1, using the same granulometry. Unlike the standard pattern spectra, these spatial pattern spectra can distinguish the three images.

\section{Discussion}

Spatial pattern spectra may form a useful supplement to ordinary pattern spectra, because of their ability to retain spatial information. This feature allows separation of images indistinguishable by standard pattern spectra. Pattern moment spectra, in particular, are easily computed concurrently with computation of the standard pattern spectrum. Post-processing of these pattern moment spectra can be done to yield a number of easily interpreted spectra, such as pattern mean, variance, skew, and kurtosis spectra, which have reduced covariance compared to the "raw" pattern moment spectra. Invariance to rotation, translation or scale change can also be achieved by post-processing [3,2].

In the binary case, Nacken's approach to computation of pattern spectra is readily extended to pattern moment spectra. The method is flexible since computing pattern moment spectra based on different granulometries reduces to computing different opening transforms.

In the future grey scale versions of these spatial pattern spectra will be developed. I expect that the efficient grey level algorithms for area and attribute pattern spectra $[8,9]$ can be adapted to spatial pattern spectra as well.

\section{References}

[1] E. J. Breen and R. Jones. Attribute openings, thinnings and granulometries. Computer Vision and Image Understanding, 64(3):377-389, 1996.

[2] J. Flusser and T. Suk. Pattern recognition by affine moment invariants. Pattern Recognition, 26:167-174, 1993.

[3] M. K. Hu. Visual pattern recognition by moment invariants. IRE Transactions on Information Theory, IT-8:179$187,1962$.

[4] C.-T. Huang, P. S. Stewart, and G. A. McFeters. The study of microbial biofilms by classical fluorescence microscopy. In M. H. F. Wilkinson and F. Schut, editors, Digital Image Analysis of Microbes, pages 411-430. John Wiley and Sons, Ltd, Chichester, UK, 1998.

[5] P. Maragos. Pattern spectrum and multiscale shape representation. IEEE Trans. Patt. Anal. Mach. Intell., 11:701-715, 1989.

[6] G. Matheron. Eléments pour une Théorie des Milieux Poreux. Masson, Paris, 1967.

[7] G. Matheron. Random Sets and Integral Geometry. John Wiley, 1975.

[8] A. Meijster and M. H. F. Wilkinson. Fast computation of morphological area pattern spectra. In Int. Conf. Image Proc. 2001, pages 668-671, 2001.

[9] A. Meijster and M. H. F. Wilkinson. A comparison of algorithms for connected set openings and closings. IEEE Trans. Patt. Anal. Mach. Intell., 24(4):484-494, 2002.

[10] P. F. M. Nacken. Image Analysis Methods Based on Hierarchies of Graphs and Multi-Scale Mathematical Morphology. $\mathrm{PhD}$ thesis, University of Amsterdam, Amsterdam, The Netherlands, 1994.

[11] A. Sofou, C. Tzafestas, and P. Maragos. Segmentation of soilsection images using connected operators. In Int. Conf. Image Proc. 2001, pages 1087-1090, 2001.

[12] L. Vincent. Morphological area openings and closings for grey-scale images. In Y.-L. O, A. Toet, D. Foster, H. J. A. M. Heijmans, and P. Meer, editors, Shape in Picture: Mathematical Description of Shape in Grey-level Images, pages 197-208. NATO, 1993.

[13] L. Vincent. Morphological grayscale reconstruction in image analysis: application and efficient algorithm. IEEE Transactions on Image Processing, 2:176-201, 1993.

[14] L. Vincent. Granulometries and opening trees. Fundamenta Informaticae, 41:57-90, 2000. 